\title{
Coherent $\pi^{0} \eta$ photoproduction on $s$-shell nuclei
}

\author{
M. Egorov and A. Fix \\ Laboratory of Mathematical Physics, Tomsk Polytechnic University, Tomsk, Russia
}

(Dated: August 24, 2021)

\begin{abstract}
Coherent photoproduction of $\pi^{0} \eta$ on the deuteron, ${ }^{3} \mathrm{He}$ and ${ }^{4} \mathrm{He}$ nuclei is considered in the energy region from threshold to the lab photon energy $E_{\gamma}=1.2 \mathrm{GeV}$. The transition amplitude is derived using impulse approximation. Effects of pion absorption are included by means of the FernbachSerber-Taylor model. Interaction of the produced $\eta$ mesons with the recoiled nucleus is taken into account for the reactions on $d$ and ${ }^{3} \mathrm{He}$. The corresponding $\eta d$ and $\eta^{3} \mathrm{He}$ scattering amplitudes are obtained as solutions of the few-body equations for $\eta N N$ and $\eta-3 N$ systems. Impact of this interaction on the differential cross section in the region of small relative $\eta$-nuclear momenta is discussed.
\end{abstract}

PACS numbers: 13.40.-f, 13.60.Le, 21.45.+v, 14.20.Gk

\section{INTRODUCTION}

Among different photoproduction channels special interest is focused today on the processes with two pseudoscalar mesons in the final state. Experimental study of $\pi \pi$ and $\pi \eta$ photoproduction on nucleons and nuclei have become an important part of the research programs of the European Laboratory for Structural Assessment (ELSA) and Mainz Microtron (MAMI) facilities [1]. In particular, the database for $\pi^{0} \eta$ photoproduction was greatly extended by the new very precise measurements, covering large region of the lab photon energy from threshold up to $3 \mathrm{GeV}$ [2 5$]$. Furthermore, a considerable amount of new data for polarization observables was reported in Refs. [6 9 ].

New experimental results generate a revival of theoretical interest to $\pi^{0} \eta$ photoproduction. Besides the most early studies of [10] a recent, rather detailed investigation of this process on a free proton was performed in [11 13]. Most of the efforts are directed towards understanding of the general dynamical properties of those $N^{*}$ and $\Delta$ resonances which are not very well seen in the reactions with a single meson and for which only a weak evidence exists [14]. Analysis of the existing data within different models has provided further insight into the details of the nucleon excitation spectrum, in particular, in the third and the fourth resonance region.

It is however clear, that systematic study of meson photoproduction requires detailed information on the same process in nuclei. Here coherent reactions are of special use. Different works clearly demonstrate their importance, especially in those cases when the production proceeds dominantly via resonance excitations. One of the main motivations for studying these reactions is to obtain information on the isotopic structure of the elementary production amplitude. Evident advantage of using light nuclei as targets is the small number of nucleons. This allows one to minimize the influence of a nuclear environment on the elementary process, on the one hand, and to adopt an accurate microscopic description of the nuclear states, on the other hand.

An important question related to $\pi^{0} \eta$ photoproduction on nuclei concerns $\eta$-nuclear interaction in the final state. Although the $\eta$-nuclear scattering problem is by itself rather many-sided, the major part of the related questions are connected to the one central point $-\eta N$ phenomenology in the low-energy regime. More specifically, the matter concerns determination of the $\eta N$ low-energy interaction parameters, primarily the scattering length $a_{\eta N}$. In the absence of the direct scattering results, final state interaction (FSI) in $\eta$ production on nuclei remains the major source of information on the $\eta N$ dynamics. Typical method of studying $\eta N$ system in these reactions follows the scheme: (i) $\eta$ production on nuclei, (ii) $\eta A$ model, (iii) $\eta N$ interaction parameters. The central point in this sequence is the $\eta$-nuclear interaction model. Since in general one intends to connect $\eta A$ properties with those of $\eta N$, the $\eta A$ model should be based on a refined microscopic approach, wherever possible, and at the same time, it should allow one to take systematically into account fundamental properties of $\eta A$ system, such as unitarity of the scattering matrix, which is especially important at low energies. Here the few-body calculations [15 20], utilizing, as a rule, separable $\eta N$ matrix, have already deserved a reputation of an effective theoretical method. Since these calculations are mostly restricted to the systems with three and four particles, by now only $\eta$ production on deuterium [21 24] and ${ }^{3} \mathrm{He}$ [25 28] is considered in detail. According to the results of Refs. [20, 25, 27], for 'reasonable' values of $\eta N$ scattering length with $R e a_{\eta N}=0.6 \pm 0.2 \mathrm{fm}$ and $\operatorname{Im} a_{\eta N}=0.3 \pm 0.1 \mathrm{fm}$, attraction strength is insufficient to generate bound states of $\eta$ with two- and three-body nuclei, so that only virtual poles in these systems appear. On the other hand, for higher values of $R e a_{\eta N}$ about $0.8 \mathrm{fm}$, the $\eta$-nuclear forces become nearly strong enough to bind the system. As a consequence, the corresponding virtual pole lies very close to the physical region, resulting in strong enhancement of the $\eta$ production cross section.

The case of ${ }^{4} \mathrm{He}$ is less clear. Firstly, the existing data [29] for the total cross section of $d d \rightarrow \eta^{4}$ He shows no threshold enhancement due to final state interaction. Furthermore, in the resent experiment of [30] no signal from the 
decay of a hypothetical $\eta$-mesic ${ }^{4} \mathrm{He}$ into $\pi^{-} p^{3} \mathrm{He}$ in the same reaction $d d \rightarrow \eta^{4} \mathrm{He}$ was detected. These results are rather surprising irrespective of existence of $\eta^{4} \mathrm{He}$ bound states. They mean that the $s$-matrix pole which in the case of $\eta^{3} \mathrm{He}$ seems to be close to the threshold energy on the Riemann surface, moves far away from this point when we turn to ${ }^{4} \mathrm{He}$. Even if one takes into account a larger number of nucleons in ${ }^{4} \mathrm{He}$ and profound increase of its density, total disappearance of a signal from $\eta^{4} \mathrm{He}$ interaction in the measured spectrum is rather unexpected. Secondly, there are still no correct few-body results for $\eta^{4} \mathrm{He}$ due to difficulty of the corresponding calculation. Less sophisticated theories, like optical model, are unable to take correctly into account important features of the low-energy $\eta$-nuclear interaction, for example, importance of the virtual target excitations between the successive scattering acts (see, e.g., [27]). Therefore, even the qualitative results obtained within this approach are unreliable. The matter is further complicated by the fact that it is the $\eta^{4} \mathrm{He}$ case, where the binding may appear. The value of the scattering length corresponding to a weakly bound or virtual state is known to be very sensitive to small variation of the potential parameters. Therefore, it may turn out that if we apply few-body formalism to $\eta^{4} \mathrm{He}$, the result will strongly depend not only on the $\eta N$ parameters, but also on the approximations used for ${ }^{4} \mathrm{He}$ states (e.g., on details of the $N N$-forces at short distances, inclusion of the repulsive core into $N N$ potential, etc.). This will lead to strong model dependence of the calculation, not to mention that the five-body scattering problem is technically very difficult by itself.

Since no microscopic $\eta A$ calculations are available for the nuclei with $A>3$, the methods allowing model independent extraction of the $\eta A$ scattering parameters directly from the measured observables are of special importance. Several steps are already done in Refs. 31 41], where information on the $\eta$-nuclear scattering from the characteristic behavior or the cross section in the region of small $\eta A$ relative energies was obtained. As a rule, the underlying method is based on the approach developed by Watson [42] and Migdal [43]. Under certain conditions this approach makes it possible to study $\eta A$ interaction directly from a distribution over the relative $\eta A$ energy. The case in point is a characteristic enhancement of the $\eta$ yield due to strong attraction between $\eta$ meson and the recoiled nucleus. Here again, coherent photoproduction of $\pi^{0} \eta$ seems to have some advantages over other reactions, like $A(p, \eta p) A$ or $A(\pi, \eta N) B$, for which one has to take into account interaction between all three final particles. Even the simplest case, when the composite nature of the nucleus is ignored (e.g., if one neglects its virtual excitations), requires three-body calculation. This problem should be much less important for $\pi^{0} \eta$ photoproduction. In this case one can safely neglect the interaction between $\eta$ and $\pi^{0}$, since in the energy region under discussion $E_{\gamma} \leq 1.5 \mathrm{GeV}$ this system does not resonate. As a result, the whole interaction process may be approximated by the sum of $\eta A$ and $\pi^{0} A$ rescatterings.

Although the reactions $A\left(\gamma, \pi^{0} \eta\right) A$ offer important advantages, up to now rather little effort has been devoted to their theoretical study. Perhaps, the major reason of this fact is difficulty of the experimental identification of these reactions due to smallness of the coherent cross section in comparison to the background quasifree process $A\left(\gamma, \pi^{0} \eta N\right)$. This situation should change with new measurements of $\pi^{0} \eta$ photoproduction on nuclei [1]. In anticipation of the new data, we present here theoretical results for $\pi^{0} \eta$ photoproduction on $s$-shell nuclei, for which we have chosen as specific examples $d,{ }^{3} \mathrm{He}$, and ${ }^{4} \mathrm{He}$.

\section{MODEL INGREDIENTS}

We consider the process

$$
\gamma\left(E_{\gamma}, \vec{k} ; \vec{\varepsilon}_{\lambda}\right)+A\left(E_{A}, \vec{Q}_{A}\right) \rightarrow \pi^{0}\left(\omega_{\pi}, \vec{q}_{\pi}\right)+\eta\left(\omega_{\eta}, \vec{q}_{\eta}\right)+A\left(E_{A}^{\prime}, \vec{Q}_{A}^{\prime}\right)
$$

where the 4-momenta of the participating particles are given in the parentheses. The calculations are performed in the laboratory frame where the initial nucleus $A$ is at rest $\left(Q_{A}=0\right)$. The circular polarization vector of the photon is denoted by $\vec{\varepsilon}_{\lambda}$ with $\lambda= \pm 1$. One of the features of the reaction (11) is rather high momentum transfer associated with a relatively large mass of the $\pi \eta$ system. This firstly results in rather low cross section (several hundreds nb) and, moreover, in sensitivity of its magnitude to details of the nuclear wave function at short internuclear distances. At the same time, this sensitivity does not necessarily mean that a refined microscopic nuclear model is required for the calculation. Indeed, according to our general notion about the coherent production, its basic mechanism, yielding the main fraction of the amplitude, is impulse approximation accompanied by the final state interaction (FSI) effects. Within this model, the unpolarized cross section is mainly governed by the nuclear form factor. The latter is free from the ambiguities of the nuclear structure and may well be described phenomenologically without resorting to complicated microscopic calculations. Furthermore, interaction of pions with nuclei in the resonance region, where its main effect is attenuation of the pion wave function inside the nucleus, may be described in terms of the pion mean free path in the nuclear matter. As for the $\eta$-nuclear interaction, which is one of the main objects of the present study, in the low energy region it is mainly determined by the long-range part of the $\eta$-nuclear wave function, and therefore, should be insensitive to the structural details of the nuclear model. Taking account of this observation, we use for the nuclear wave functions the phenomenological models, which reproduce the corresponding formfactors up to the values of momentum transfer, which are characteristic for $\pi \eta$ production in the second and the third resonance region. 

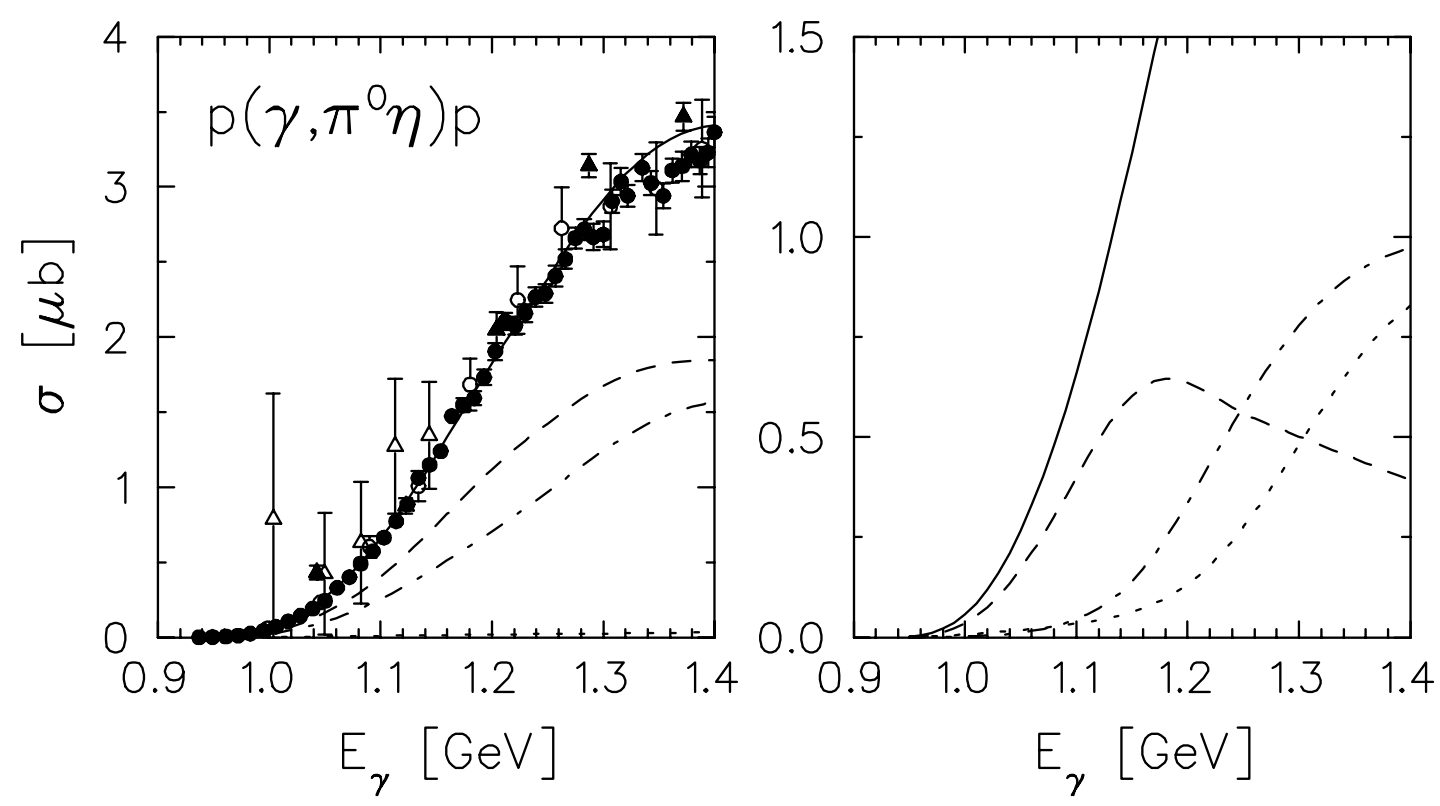

FIG. 1: Total cross section for $\gamma p \rightarrow \pi^{0} \eta p$ calculated using the isobar model of Ref. 12] (solid curve on both panels). On the left panel, the dash-dotted and the dashed curves correspond to the spin-flip part $\vec{K}$ and the spin independent part $L$ in Eq. (2). The dotted curve shows the contribution of the isoscalar part $A^{(0)}$ of the amplitude (8). The data are from Ref. [2] (empty triangles), 3] (empty circles), 4] (filled triangles), and [5] (filled circles). On the right panel, the dashed and dash-dotted curves show the contribution from the resonances $D_{33}(1700)$ and $D_{33}(1940)$, respectively. The dotted curve is the combined contribution of the remaining resonances $\left(P_{33}(1600), P_{31}(1750), F_{35}(1905), P_{33}(1920)\right)$ and the Born terms.

For the deuteron we take the wave function of the Bonn potential (full model) [44]. For ${ }^{3} \mathrm{He}$ target the separable parametrization from [45] is employed, and for ${ }^{4} \mathrm{He}$ we adopt Fourier transform of the $r$-space wave function from [46].

The elementary operator has the well known form

$$
t_{\gamma N \rightarrow \pi \eta N}=L+i \vec{K} \cdot \vec{\sigma}
$$

reflecting the general spin structure of photoproduction of pseudoscalar mesons on spin $1 / 2$ fermions. Here we used the isobar model from Ref. [12] (first solution). Apart from the dominant $D_{33}(1700)$ and $D_{33}(1940)$ resonances, the model [12] also contains the positive parity states $P_{33}(1600), P_{31}(1750), F_{35}(1905), P_{33}(1920)$ and relatively small admixture of the Born terms. The amplitude is diagrammatically presented in Fig. 2 of Ref. [12]. The parameters were fitted to the experimental angular distributions of pions and $\eta$ mesons in $\gamma p \rightarrow \pi^{0} \eta p$. In Fig.1 we show the total cross section of this reaction. The contributions of different terms are separately presented on the right panel. According to the calculation, in the region up to $E_{\gamma}=1.2 \mathrm{GeV}$ the major fraction of the cross section is provided by the resonance $D_{33}(1700)$.

On the left panel of Fig.1 1 we also plotted the components $\sigma_{K}$ and $\sigma_{L}$ of the cross section coming from the spin-flip and spin independent part of the operator (2). Neglecting all terms apart from $D_{33}(1700)$ one can obtain for the ratio of $\sigma_{K}$ to $\sigma_{L}$

$$
\frac{\sigma_{K}}{\sigma_{L}}=\frac{1}{2}+\frac{1}{2}\left(\frac{3-\sqrt{3} a}{1+\sqrt{3} a}\right)^{2},
$$

where $a$ is the ratio of $3 / 2$ to $1 / 2$ helicity amplitudes of the $D_{33}(1700)$ resonance

$$
a=A_{3 / 2} / A_{1 / 2} .
$$

In the model [12] this parameter changes from 0.9 to 1.1 in the region $E_{\gamma} \leq 1.2 \mathrm{GeV}$, where $D_{33}(1700)$ dominates (see Fig. 6 in [12]). Therefore, at these photon energies the ratio (3) remains almost constant and is equal to $0.60 \pm 0.04$, so that the components $\sigma_{K}$ and $\sigma_{L}$ are comparable, as may also be seen from Fig.1. 
Within the impulse approximation the amplitude on a nucleus is proportional to that on a single nucleon sandwiched between the states including the initial and the final nucleus in the ground state with the spin $J$

$$
T_{\lambda M M^{\prime}}=A\left\langle\vec{q}_{\eta}, \vec{q}_{\pi} ; J M^{\prime}\left|t_{\gamma N \rightarrow \pi \eta N}(\omega)\right| \vec{k}, \lambda ; J M\right\rangle,
$$

with $A$ denoting the number of nucleons in the target. The quantity $\omega$ in (5) has a meaning of the invariant $\gamma N$ energy when the nucleon is on the mass shell. To take properly into account the Fermi motion effect, which should be important in the resonance region, we use the prescription [47, 48], in which the elementary operator is frozen at the average effective nucleon momentum in the laboratory system

$$
\vec{p}_{i}=\left\langle\vec{p}_{i}\right\rangle=-\frac{A-1}{2 A} \vec{Q},
$$

where $\vec{Q}=\vec{k}-\vec{q}_{\pi}-\vec{q}_{\eta}$ is the momentum transferred to the nucleus and $A$ is the nuclear mass number. This choice is compatible with the requirements of energy and momentum conservation together with the on-mass-shell conditions for the nucleon both in the initial and the final state.

The unpolarized cross section of the reaction (11) is proportional to the square of the amplitude (5) averaged over the spin states

$$
\frac{d \sigma}{d \Omega_{\pi} d \omega_{\pi} d \Omega_{\eta}^{*}}=\frac{1}{(2 \pi)^{5}} \frac{E_{A}^{\prime} q_{\pi} q_{\eta}^{*}}{8 E_{\gamma} \omega_{\eta A} W} \frac{1}{2(2 J+1)} \sum_{\lambda M M^{\prime}}\left|T_{\lambda M M^{\prime}}\right|^{2}
$$

where the total $\eta A$ energy $\omega_{\eta A}$, as well as the $\eta$ momentum $\vec{q}_{\eta}^{*}$ and the corresponding solid angle $\Omega_{\eta}^{*}$ are calculated in the $\eta A$ center-of-mass (c.m.) frame.

Of primary importance for the coherent reaction is a relative contribution of the transitions with isospins $I=1 / 2$ and $I=3 / 2$. Since $\eta$ is an isoscalar particle, the isospin structure of the operator (2) is similar to that of single pion photoproduction. In particular, for $\pi^{0} \eta$ we have

$$
t_{\gamma N \rightarrow \pi \eta N}=A^{(0)} \tau_{3}+\frac{1}{3} A^{(1 / 2)}+\frac{2}{3} A^{(3 / 2)},
$$

where $\tau_{3}$ is the third component of the nucleon isospin operator $\vec{\tau}$. The amplitudes $A^{(1 / 2)}$ and $A^{(3 / 2)}$ in (8) are related to the final $\pi^{0} \eta N$ state with the isospin $I=1 / 2$ and $I=3 / 2$, respectively. The isoscalar amplitude $A^{(0)}$ leads only to the states with isospin $1 / 2$. According to the analyses of [10 12] in the region $E_{\gamma}=1-1.2 \mathrm{GeV}$ the process (2) is dominated by excitation of $\Delta$-like resonances, so that the role of the $A^{(0)}$ and $A^{(1 / 2)}$ components is small in the energy region considered. Therefore, the resulting elementary cross section is practically the same for proton and neutron targets. For the reactions on nuclei this means that the effect of coherence is maximal and the cross section does not depend on the isospin of the target.

To take into account interaction between the emitted pion and the final nucleus we used a simplified model in which this interaction is described in terms of classical propagation of a pion in a nuclear matter. In the resonance region the major impact of a nucleus is attenuation of the pion beam due to absorption. Apart from the true absorption on nucleon pairs, the inelastically scattered pions, which in fact contribute to the incoherent cross section, are also treated as if they are absorbed. Additional interaction effect, which however should be less important in the region considered, comes from the modification of the pion wave number in a nuclear medium, which in particular results in changing diffraction patterns which are characteristic for coherent pion photoproduction in the resonance region. Here we neglect the last effect and take into account only absorption of the produced pions using the simple prescription. Namely, the cross section is multiplied by the energy dependent damping factor, which was calculated as follows. The pion wave function inside the nucleus was taken in the form used by Fernbach, Serber, and Taylor [49] for neutron interactions in nuclei:

$$
\phi_{\vec{q}_{\pi}}^{(-)}(\vec{r})=\exp \left(-i \vec{q}_{\pi} \cdot \vec{r}\right) D(\vec{r}), \quad D(\vec{r})=\exp (-l(\vec{r}) / 2 \lambda),
$$

where the damping factor $D(\vec{r})$ depends on the distance $l(\vec{r})$, measured along the classical trajectory of a meson between the point where it was produced and the point where it escaped from the nucleus. The optical properties of the nuclear environment are determined by the mean free path $\lambda$ of a pion. It can be expressed in terms of the $\pi N$ scattering cross section $\sigma_{\pi N}$ averaged over protons and neutrons as

$$
\lambda=\frac{1}{\rho \sigma_{\pi N}},
$$



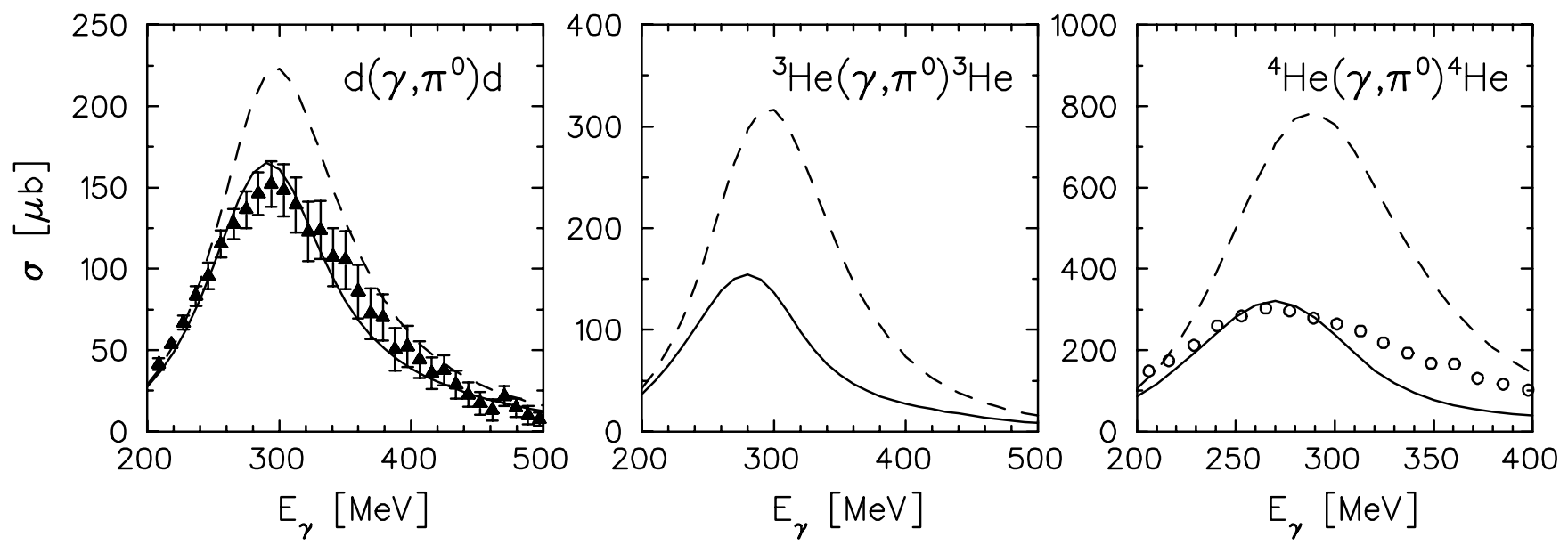

FIG. 2: Total cross section for coherent $\pi^{0}$ photoproduction on $d,{ }^{3} \mathrm{He}$, and ${ }^{4} \mathrm{He}$. The solid (dashed) curves are calculated using impulse approximation with (without) pion absorption. The elementary $\gamma N \rightarrow \pi^{0} N$ amplitude is taken from the MAID2007 model [51]. The data are from [52] (triangles) and [53] (circles).

where $\rho$ is the nuclear density. For simplicity we take the damping factor $D(\vec{r})$ out of the matrix element at a mean value

$$
\overline{D(\vec{r})}=\frac{1}{A} \int_{V} D(\vec{r}) \rho(r) d^{3} r
$$

where $V$ is the nuclear volume. For the nuclear density $\rho(r)$ in (10) and (11) a simple hard sphere form

$$
\begin{aligned}
\rho(r) & =\frac{3 A}{4 \pi R^{3}}, & & r<R, \\
& =0, & & r>R,
\end{aligned}
$$

was taken, where for $R$ we used the r.m.s. radius $R=\sqrt{\left\langle r^{2}\right\rangle}$. Then the absorption effect results in suppressing the cross section by the factor (see also [50])

$$
\overline{D(\vec{r})}^{2}=\frac{9}{4 x^{2}}\left\{1-\frac{2}{x^{2}}\left[1-(1+x) e^{-x}\right]\right\}^{2}, \quad x=\frac{R}{\lambda} .
$$

In Fig. 2 we show our results for coherent single $\pi^{0}$ photoproduction on all three nuclei in the first resonance region. As one can see, for the reaction on $d$ and ${ }^{4} \mathrm{He}$ the method provides the required suppression in the region of maximum at $E_{\gamma} \approx 280 \mathrm{MeV}$. Above $E_{\gamma} \approx 320 \mathrm{MeV}$ the calculated cross section underestimates the experimental results. However, in view of extreme simplicity of our model the agreement with the data is quite reasonable.

Contrary to the pion case, interaction in the $\eta$-nucleus system, where the major role is played by the strong $s$-wave attraction, is mostly important at lower relative energies. This well known property is also observed in the $p d \rightarrow p \eta d$ reaction [28, 33] where it leads to a pronounced peak in the distribution over the relative $\eta$-nuclear energy $E$ in the region of small $E$ values. To extract the $\eta A$ interaction parameters, one assumes that this FSI effect is independent on the $\eta$ production mechanism, and, therefore, can be unambiguously isolated. In fact, this assumption is justified only if the following conditions are fulfilled [42, 43] : (i) the driving reaction mechanism $\left(\pi^{0} \eta\right.$ photoproduction in our case) is of short-range nature, i.e. its effective radius is essentially smaller than the characteristic range of $\eta A$ forces, and (ii) attraction between the particles is comparatively strong and is characterized by low relative momentum, so that it acts during a sufficiently long time. For the reactions with more than two strongly interacting particles in the final state the third obvious condition should be added: (iii) other particles having high velocities, quickly escape the region in which the production mechanism works, and thus have little effect on the interacting pair. From the three conditions above the first two seem to be fairly well satisfied in our case. Indeed, the smallness of the effective range of an interaction responsible for $\pi \eta$ production is ensured by rather large momentum transfer. Strong $\eta A$ attraction is due to the nearby pole in the corresponding $s$-wave amplitudes. The third condition is fulfilled due to the small pion mass, which results in rather high velocity of the produced pion, so that in the major fraction of the reaction events it is at a distance well removed from the $\eta A$ pair. This latter aspect is considered in the next section in more detail. 
TABLE I: Parameters for $\eta d$ and $\eta^{3}$ He low-energy scattering. The values are obtained by fitting the scattering amplitudes, calculated within the few-body formalism described in Refs. 19] and [27]. For orientation the corresponding values of $\eta N$ scattering lengths are also given.

\begin{tabular}{c|c|cc|cc}
\hline & $a_{\eta N}[\mathrm{fm}]$, Ref. & $a_{\eta d}[\mathrm{fm}]$ & $r_{0 \eta d}[\mathrm{fm}]$ & $a_{\eta^{3} H e}[\mathrm{fm}]$ & $r_{0 \eta^{3} H e}[\mathrm{fm}]$ \\
\hline 1 & $0.50+i 0.33,[54]$ & $1.232+i 1.110$ & $2.429-i 1.037$ & $1.866+i 2.752$ & $1.934-i 0.532$ \\
2 & $0.75+i 0.27,[55]$ & $2.221+i 1.153$ & $1.870-i 0.471$ & $4.199+i 4.817$ & $1.442-i 0.139$ \\
3 & $1.03+i 0.41,[56]$ & $3.318+i 2.648$ & $1.818-i 0.456$ & $-3.767+i 9.362$ & $1.631-i 0.187$ \\
\hline
\end{tabular}

To take into account the $\eta$-nuclear FSI, we add to the impulse approximation $T^{I A}$ the interaction term $T^{\eta A}$

$$
T=T^{I A}+T^{\eta A},
$$

with

$$
T^{\eta A}=\int T^{I A}\left(\vec{q}^{\prime}\right) G_{\eta A}\left(q_{\eta}^{*}, q^{\prime}\right) t_{\eta A}\left(q_{\eta}^{*}, q^{\prime} ; \theta\right) \frac{d^{3} q^{\prime}}{(2 \pi)^{3}} .
$$

Here, $t_{\eta A}\left(q_{\eta}^{*}, q^{\prime} ; \theta\right)$ with $\cos \theta=\hat{q}_{\eta}^{*} \cdot \hat{q}^{\prime}$ is the half-off-shell $\eta A$-matrix. In the on-shell region it is related to the $\eta A$ scattering amplitude as

$$
t_{\eta A}\left(q_{\eta}^{*}, q_{\eta}^{*} ; \theta\right)=-\frac{2 \pi}{\mu_{\eta A}} f_{\eta A}\left(q_{\eta}^{*}, \theta\right)
$$

with $\eta$-nuclear reduced mass denoted by $\mu_{\eta A}$. The function $G_{\eta A}$ in Eq. (15) stands for the $\eta A$ propagator in the momentum representation

$$
G_{\eta A}\left(q_{\eta}^{*}, q^{\prime}\right)=\frac{1}{\omega_{\eta A}-q^{\prime 2} / 2 \mu_{\eta A}+i \epsilon} .
$$

Due to the $s$-wave character of the main $\eta N$ interaction mechanism, excitation of the resonance $S_{11}(1535)$, practically the whole amplitude $f_{\eta A}$ is saturated by its $s$-wave part $f_{0}$ :

$$
f_{\eta A}\left(q_{\eta}^{*}, \theta\right) \approx f_{0}\left(q_{\eta}^{*}\right) .
$$

We used three different sets of $\eta N$ scattering matrix parameters which give $\eta A$ scattering lengths and effective ranges listed in Table [. For orientation also the corresponding values of $\eta N$ scattering length $a_{\eta N}$ are presented. Since there is no microscopic calculation for $\eta^{4} \mathrm{He}$, the $\eta$-nuclear interaction was taken into account only for the reactions on a deuteron and ${ }^{3} \mathrm{He}$, whereas for ${ }^{4} \mathrm{He}$ only absorption of the produced pions was included.

\section{DISCUSSION OF THE RESULTS}

We would like to start our discussion from the total cross section presented in Fig. 3. As noted above, the cross section value is independent of the nuclear isospin and should be mostly determined by the spin of the target and its density. In particular, the cross section turns out to be rather sensitive to the details of the target wave function. For example, if one neglects the deuteron $d$-wave component, the total cross section for $d\left(\gamma, \pi^{0} \eta\right) d$ is reduced by about $30 \%$ at $E_{\gamma}=1 \mathrm{GeV}$. Furthermore, since, as discussed above, the contributions of the spin-flip and spin independent part in $\pi^{0} \eta$ photoproduction are comparable, the nuclear cross section strongly depends on the nuclear spin. The interplay between the nuclear fromfactor at high momentum transfer and the spin structure of the production matrix element leads to nontrivial dependence of the cross section on the choice of the target. As we can see from Fig. 3, without FSI the deuteron cross section turns out to be almost twice as large as that on ${ }^{3} \mathrm{He}$.

Since for $\pi$-nucleus interaction we take into account only absorption, the only influence of $\pi A$ FSI is attenuation of the cross section. Clearly, it should increase with increasing number of nucleons and increasing the nuclear density. 

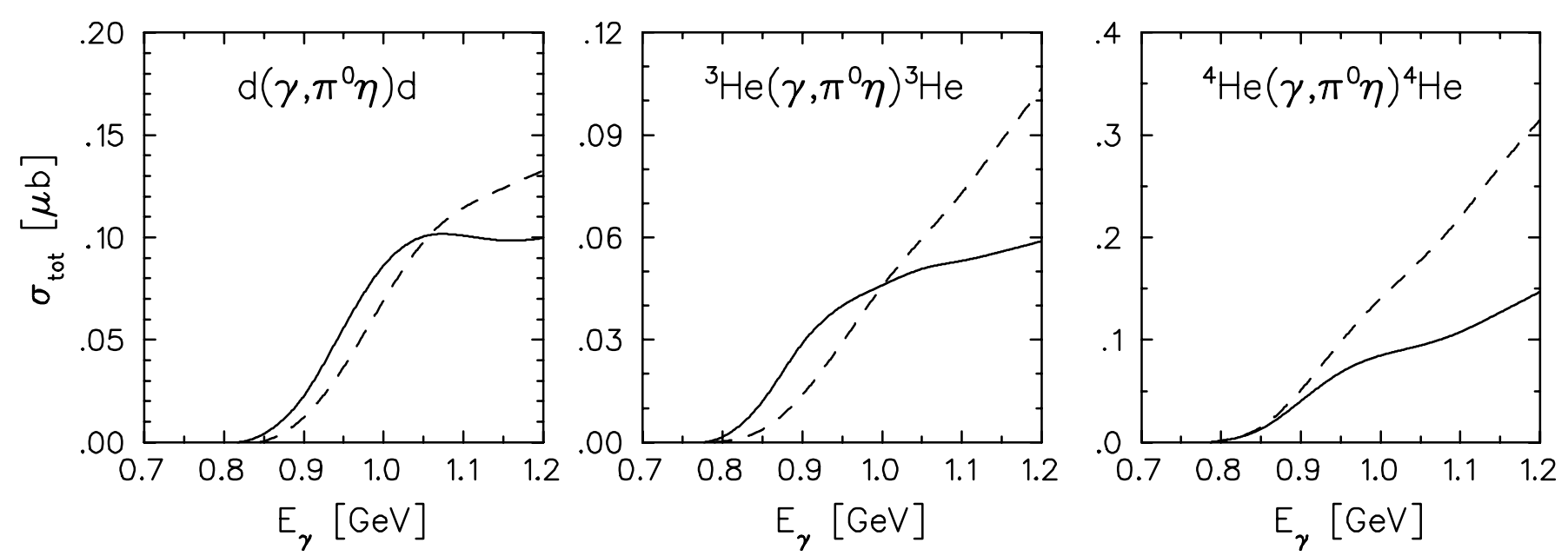

FIG. 3: Total cross section for $\pi^{0} \eta$ photoproduction on the deuteron, ${ }^{3} \mathrm{He}$ and ${ }^{4} \mathrm{He}$. The solid (dashed) curves are obtained with (without) inclusion of interaction in the final state. For $\eta A$ interaction the set of parameters 2 in Table 1 was used. In the case of ${ }^{4} \mathrm{He}$ only pion absorption was taken into account.

This means that among the three nuclei considered here the largest effect should be observed for ${ }^{4}$ He. Furthermore, absorption is known to be especially important in the region of $\Delta(1232)$, where $\pi A$ scattering becomes highly inelastic, leading for heavier nuclei to the so-called surface production mechanism. In our case it is responsible for a significant reduction of the total cross section especially above $E_{\gamma}=1 \mathrm{GeV}$.

As already noted above, an important feature of the reaction (11) is that the time which the produced pion spends in the interaction region is short in comparison to that for the $\eta$ meson. To demonstrate this feature we present in Fig. 4 the distribution of the cross section for ${ }^{3} \mathrm{He}\left(\gamma, \pi^{0} \eta\right)^{3} \mathrm{He}$ over the relative velocity in the $\eta^{3} \mathrm{He}$ and $\pi^{3} \mathrm{He}$ subsystems. The velocity was calculated in the corresponding $\eta A$ and $\pi A$ center-of-mass (c.m.) frames as

$$
v_{m A}=\frac{v_{m}^{*}+v_{A}^{*}}{1+v_{m}^{*} v_{A}^{*}}=\frac{\lambda^{1 / 2}\left(\omega_{m A}^{2}, M_{m}^{2}, M_{A}^{2}\right)}{\omega_{m A}^{2}-M_{m}^{2}-M_{A}^{2}}, \quad m \in\{\pi, \eta\},
$$

where $v_{m}^{*}$ and $v_{A}^{*}$ are the c.m. velocities of the meson $m$ and the residual ${ }^{3}$ He nucleus, and the triangle function $\lambda$ is defined as

$$
\lambda(x, y, z)=(x-y-z)^{2}-4 y z
$$

As one can see, the maximum in the distribution over $v_{\pi A}$ is shifted to much higher values with respect to the maximum of $d \sigma / d v_{\eta A}$. The corresponding average values of $v_{m A}$ are $v_{\eta A}=0.38 \mathrm{c}$ and $v_{\pi A}=0.83 \mathrm{c}$.

Using the distribution in Fig. 4 one can estimate the characteristic time the meson $m$ takes to propagate a scattering center of radius $R$ as

$$
t_{m}=\frac{R+1 / q_{m}^{*}}{v_{m A}}+Q_{m}
$$

where the wavelength $1 / q_{m}^{*}$ takes into account the wave properties of a particle and $Q_{m}$ is a time delay due to attraction. Taking $v_{\pi A}=0.83 \mathrm{c}, q_{\pi}^{*}=190 \mathrm{MeV}$ (corresponds to $v_{\pi A}$ ), $R=2 \mathrm{fm}$, and $Q_{\pi}=1 / \Gamma_{\Delta} \approx 1 / 120 \mathrm{MeV}^{-1}$ one obtains for the pion $t_{\pi} \approx 1.62 \cdot 10^{-23} \mathrm{~s}$.

To calculate $Q_{\eta}$ one can use the Eisenbud-Wigner formula [57]

$$
Q_{\eta}=2 \frac{d}{d E} \delta_{0}(E)
$$

where $\delta_{0}(E)$ is the phase shift of the $s$-wave $\eta A$ scattering, for which one can take

$$
\delta_{0}(E) \approx \operatorname{Re} a_{\eta A} \sqrt{2 \mu_{\eta A} E} .
$$

The relative $\eta A$ energy $E$ is determined as

$$
E=\omega_{\eta A}-M_{\eta}-M_{A}
$$




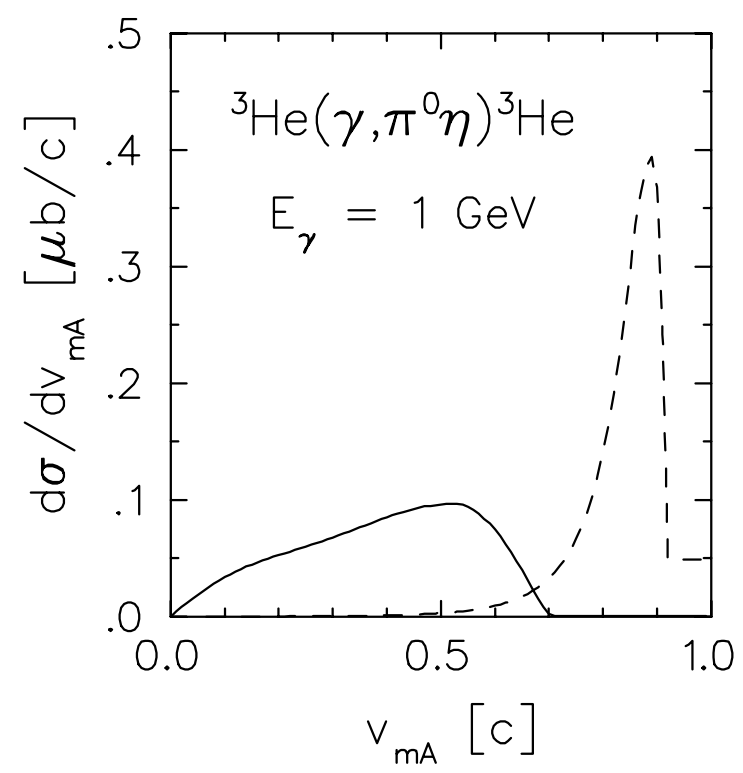

FIG. 4: Distribution of the cross section for ${ }^{3} \mathrm{He}\left(\gamma, \pi^{0} \eta\right)^{3} \mathrm{He}$ over the relative velocity in the $\eta^{3} \mathrm{He}$ (solid line) and $\pi^{3} \mathrm{He}(\mathrm{dashed}$ line) subsystems.

where $M_{A}$ is the mass of the nucleus. Taking $R e a_{\eta^{3} \mathrm{He}}=4 \mathrm{fm}$ from Table $\square$ and $E=38 \mathrm{MeV}$ (corresponds to the average relative velocity $v_{\eta A}=0.38 \mathrm{c}$ ), one obtains for the time delay $Q_{\eta}=6.67 \cdot 10^{-23} \mathrm{~s}$, so that the resulting value of $t_{\eta}$ (21) turns out to be $9.34 \cdot 10^{-23} \mathrm{~s}$, almost six times larger than $t_{\pi}$. This result supports our intuitive assumption that the pion tends to quickly escape the interaction region and its presence should have little effect on the $\eta A$ interaction.

To demonstrate the role of $\eta$-nuclear interaction, we show in Figs. 5 and 6 the distribution over the relative $\eta A$ energy $E$. As expected, the spectrum rises rapidly from zero and exhibits a peak very close to the lower limit $E=0$. On the whole, inclusion of $\eta A$ FSI enhances the $\eta$ yield. The resulting total cross in Fig. 3 is visibly increased due to $\eta A$ attraction in the region up to $E_{\gamma}=1 \mathrm{GeV}$. At higher energies, the pion absorption takes over leading to the eduction of the cross section.

In the region of low values of the relative $\eta A$ momenta $q_{\eta}^{*} R \ll 1$, the shape of the spectrum close to the peak may be described in a simple manner as [42, 43]

$$
\frac{d \sigma}{d E}(E) \sim P(E)\left|f_{0}(E)\right|^{2},
$$

where $f_{0}(E)$ is the $\eta A$ scattering amplitude and $P(E)$ is the reaction phase space. Using the effective range formula

$$
q_{\eta}^{*} \cot \delta_{0}=\frac{1}{a}+\frac{r_{0}}{2} q_{\eta}^{* 2}
$$

one can expand the ratio $P(E) / \sigma(E)$ in powers of the momentum $q_{\eta}^{*}$

$$
\frac{P(E)}{d \sigma / d E} \sim \frac{1}{\left|f_{0}(E)\right|^{2}}=q_{\eta}^{* 2}\left|\cot \delta_{0}-i\right|^{2}=\sum_{n=0}^{4} C_{n} q_{\eta}^{* n} .
$$

Since the scattering length $a_{\eta A}$ has nonzero imaginary part, the expansion (27) contains odd powers of $q_{\eta}^{*}$. In particular, the linear term $C_{1} q_{\eta}^{*}$ is proportional to

$$
C_{1}=-2 \frac{\operatorname{Im} a_{\eta A}}{\left|a_{\eta A}\right|^{2}} .
$$

Information on the relative value of the imaginary part of the scattering length is of special interest in the case of $\eta^{3} \mathrm{He}$ interaction in view of existing discrepancy between the theoretical values of $a_{\eta^{3} \mathrm{He}}$ and the analysis of measurements of $p d \rightarrow \eta^{3} \mathrm{He}$ in Ref. [34 36]. According to the latter results $I m a_{\eta^{3} \mathrm{He}}$ is about 9 times smaller than $R e a_{\eta^{3} \mathrm{He}}$, being in disagreement with the theoretical predictions of [20, 22, 25]. Such a strong suppression of the $\eta N$ inelasticity in 

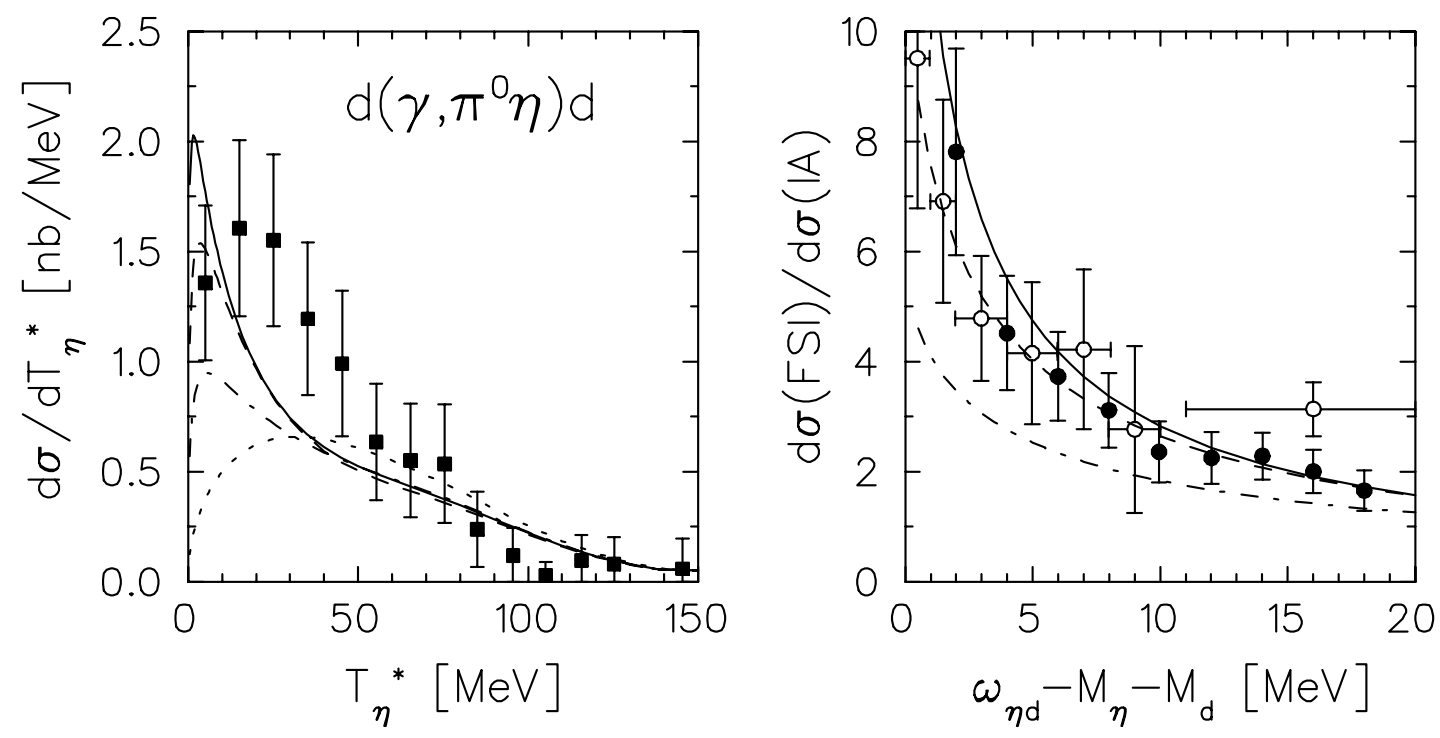

FIG. 5: Left: kinetic energy spectrum of $\eta$-mesons in the reaction $d\left(\gamma, \pi^{0} \eta\right) d$ averaged over the energy range $E_{\gamma}=0.9-1.1$ $\mathrm{GeV}$. The dotted curve is calculated without $\eta d$ interaction. The dash-dotted, dashed, and the solid curves are obtained with the sets 1,2, and 3 of $\eta A$ scattering parameters listed in Table [] The filled squares are the preliminary data from Ref. [1]. Right: ratio of the FSI to IA cross sections plotted against the relative $\eta d$ energy. Notations of the curves as on the left panel. Empty and filled circles show the $p n \rightarrow \eta d$ and $p d \rightarrow \eta p d$ cross sections from Refs. [32] and [33], respectively, divided by the arbitrarily normalized phase space.

a nucleus is also in contradiction to the intuitive expectation that with increasing number of nucleons the inelastic effects in $\eta A$ interaction should become more and more important, so that for heavier nuclei the enhancement effect due to $\eta A$ attraction is completely overshadowed by the absorption and is practically invisible [59, 60].

Also desirable are measurements of the spectrum in the reaction $\gamma^{4} \mathrm{He} \rightarrow \pi^{0} \eta^{4} \mathrm{He}$ in the region of low $\eta^{4} \mathrm{He}$ relative energies. As already noted in Introduction, an enhancement effect due to $\eta$-nuclear attraction which is rather well seen in the reactions $\gamma^{3} \mathrm{He} \rightarrow \eta^{3} \mathrm{He}$ and $p d \rightarrow \eta^{3} \mathrm{He}$ was not observed in the case of ${ }^{4} \mathrm{He}$ [29, 30]. It is therefore important to prove, whether also the peak in the distribution over the relative energy $\eta^{4} \mathrm{He}$ in the above reaction will disappear or at least will be much less pronounced in comparison to that observed on $d$ and ${ }^{3} \mathrm{He}$.

\section{CONCLUSION}

We considered several aspects related to the coherent photoproduction of $\pi^{0} \eta$ pairs on the $s$-shell nuclei. As is discussed in Introduction, these reactions have some clear advantages making them preferable to corresponding processes with hadrons as probes. Because of relative weakness of the electromagnetic interaction, photo-induced reactions are known to furnish a special opportunity to study effects of interaction in the final state. Furthermore, the underlying elementary process $\gamma N \rightarrow \pi^{0} \eta N$ seems to be under control, in the sense that the results of different analyses [10 12] of the existing data agree with each other. This is in contrast to the reactions $p d \rightarrow \eta p d$ or $p d \rightarrow \eta^{3} \mathrm{He}$ where the driving mechanism is still not completely understood [31, 61, 62]. Furthermore, due to smallness of the pion mass it tends to escape the interaction region with high velocity, and the major fraction of the production events correspond to low relative velocity between $\eta$ and the recoiled nucleus. This allows a cleaner way to study $\eta$-nuclear interaction, in comparison to $p d \rightarrow \eta p d$ where $p d$ interaction in the final state should always strongly affect the interaction between $\eta$ and the deuteron. Therefore, measurements of these reactions may be an additional important source of information on $\eta N$ low-energy dynamics.

One of the innovations of the present paper is a study of dependence of the total cross section on the spin and isotopic spin of the target. Since $\pi^{0} \eta$ photoproduction seems to be dominated by the $D_{33}$ wave, among the $s$-shell nuclei the largest cross section is predicted for ${ }^{4} \mathrm{He}$ whereas for ${ }^{3} \mathrm{He}$ it appears to be twice as small as for the deuteron.

We analyzed the effects of final state interaction in the region of low $\eta A$ relative energies. In the simplest case when the pole in the amplitude is close to the zero energy, measurement of the distribution over the relative $\eta A$ energy may be utilized to estimate the relative value of the imaginary part of the scattering length $a_{\eta} A$ using a simple expression for the linear term in the polynomial ansatz (27). This information is clearly important for our understanding of the 

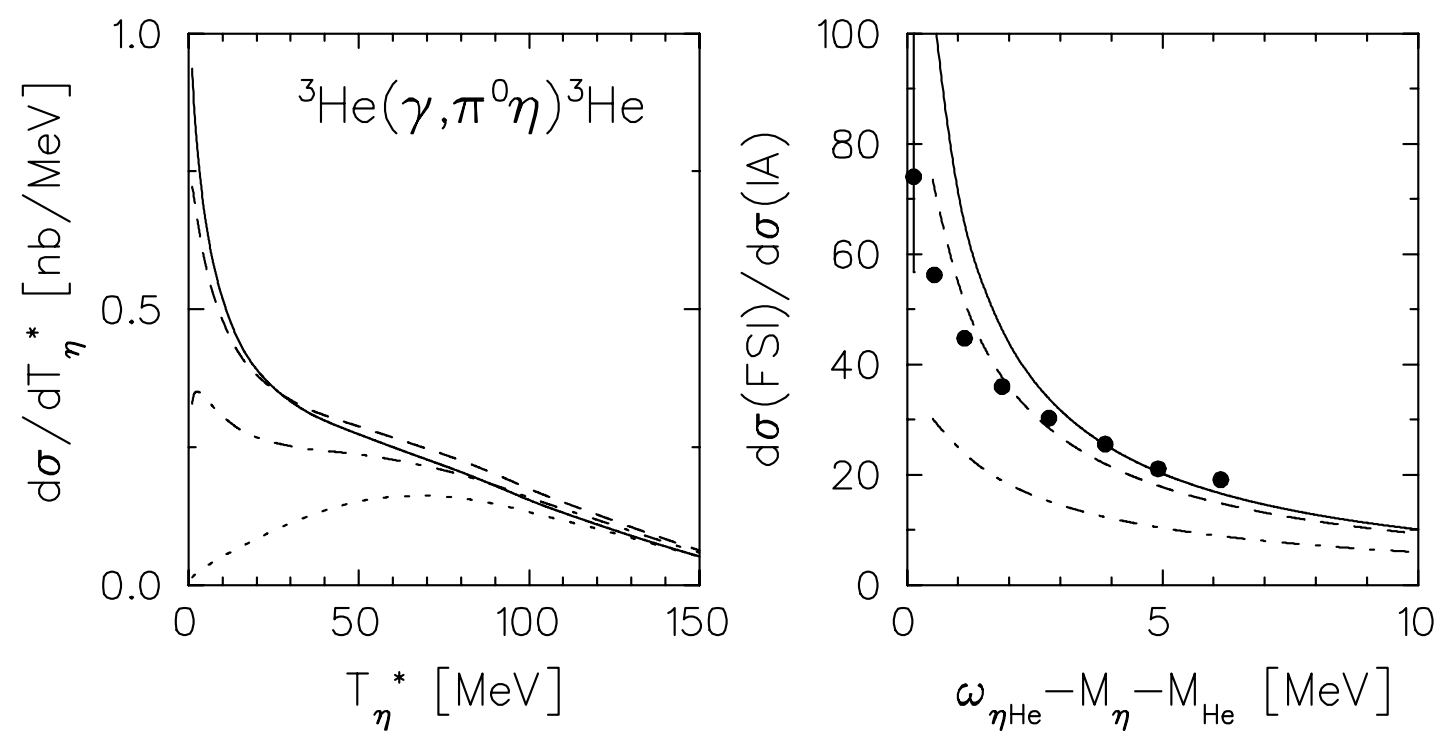

FIG. 6: Same as in Fig. 5 for ${ }^{3} \mathrm{He}\left(\gamma, \pi^{0} \eta\right)^{3} \mathrm{He}$. The points on the right panel show the $p d \rightarrow \eta^{3}$ He cross section from Ref. [58] divided by the phase space. The normalization of the data is arbitrary.

role of inelasticity in $\eta A$ low energy interaction.

\section{Acknowledgment}

Valuable discussions with Christoph Hanhart are much appreciated. We also acknowledge support from the RF Federal programm "Kadry" (contract 14.B37.21.0786), MSE Program 'Nauka' (contract 1.604.2011) and from the Ministry of education and science of Russian Federation (project 16.740.11.0469).

[1] B. Krusche, Eur. Phys. J. ST 198 (2011) 199 arXiv:1110.0192 [nucl-ex]].

[2] T. Nakabayashi, H. Fukasawa, R. Hashimoto, T. Ishikawa, T. Iwata, H. Kanda, J. Kasagi and T. Kinoshita et al., Phys. Rev. C 74 (2006) 035202.

[3] J. Ajaka, Y. Assafiri, O. Bartalini, V. Bellini, S. Bouchigny, M. Castoldi, A. D'Angelo and J. P. Didelez et al., Phys. Rev. Lett. 100 (2008) 052003.

[4] I. Horn et al. [CB-ELSA Collaboration], Phys. Rev. Lett. 101 (2008) 202002 arXiv:0711.1138 [nucl-ex]].

[5] V. L. Kashevarov et al. [Crystal Ball at MAMI and TAPS and A2 Collaborations], Eur. Phys. J. A 42 (2009) 141 arXiv:0901.3888 [hep-ex]].

[6] E. Gutz et al. [CBELSA Collaboration], Eur. Phys. J. A 35 (2008) 291.

[7] E. Gutz et al. [CBELSA and TAPS Collaborations], Phys. Lett. B 687 (2010) 11 arXiv:0912.2632 [nucl-ex]].

[8] E. Gutz [CBELSA-TAPS Collaboration], AIP Conf. Proc. 1257 (2010) 581.

[9] V. L. Kashevarov et al. [Crystal Ball at MAMI, TAPS, and A2 and TAPS and A2 Collaborations], Phys. Lett. B 693 (2010) 551 arXiv:1009.4093 [nucl-ex]].

[10] M. Doring, E. Oset and D. Strottman, Phys. Rev. C 73 (2006) 045209 nucl-th/0510015.

[11] I. Horn, et al., Eur. Phys. J. A 38 (2008) 173 arXiv:0806.4251 [nucl-ex]].

[12] A. Fix, V. L. Kashevarov, A. Lee and M. Ostrick, Phys. Rev. C 82 (2010) 035207 arXiv:1004.5240 [nucl-th]].

[13] M. Doring, E. Oset and U. -G. Meissner, Eur. Phys. J. A 46 (2010) 315 arXiv:1003.0097 [nucl-th]].

[14] J. Beringer et al. [Particle Data Group Collaboration], Phys. Rev. D 86 (2012) 010001.

[15] N. V. Shevchenko, S. A. Rakityansky, S. A. Sofianos, V. B. Belyaev and W. Sandhas, Phys. Rev. C 58 (1998) R3055.

[16] A. Deloff, Phys. Rev. C 61 (2000) 024004.

[17] H. Garcilazo and M. T. Pena, Phys. Rev. C 61 (2000) 064010 nucl-th/0002056.

[18] S. Wycech and A. M. Green, Phys. Rev. C 64 (2001) 045206 nucl-th/0104053.

[19] A. Fix and H. Arenhovel, Nucl. Phys. A 697 (2002) 277 nucl-th/0104032.

[20] A. Fix and H. Arenhovel, Phys. Rev. C 66 (2002) 024002 nucl-th/0202080].

[21] A. Fix and H. Arenhovel, Phys. Lett. B 492 (2000) 32 nucl-th/0008031. 
[22] N. V. Shevchenko, V. B. Belyaev, S. A. Rakityansky, S. A. Sofianos and W. Sandhas, Nucl. Phys. A 689 (2001) 383 nucl-th/0012041.

[23] H. Garcilazo and M. T. Pena, Phys. Rev. C 66 (2002) 034606.

[24] A. Fix and H. Arenhovel, Eur. Phys. J. A 19 (2004) 275 nucl-th/0305098.

[25] S. Wycech, A. M. Green, and J. A. Niskanen, Phys. Rev. C 52 (1995) 544.

[26] N. V. Shevchenko, V. B. Belyaev, S. A. Rakityansky, S. A. Sofianos and W. Sandhas, Nucl. Phys. A 699 (2002) 165.

[27] A. Fix and H. Arenhoevel, Phys. Rev. C 68 (2003) 044002 nucl-th/0302050.

[28] N. J. Upadhyay, K. P. Khemchandani, B. K. Jain and N. G. Kelkar, Phys. Rev. C 75 (2007) 054002

[29] R. Frascaria, F. Roudot, M. A. Duval, R. Wurzinger, W. Spang, J. Ernst, F. Hinterberger and R. Jahn et al., Phys. Rev. C 50 (1994) 537.

[30] P. Adlarson et al. [WASA-at-COSY Collaboration], arXiv:1301.0843 [nucl-ex].

[31] J. F. Germond and C. Wilkin, J. Phys. G 15 (1989) 437.

[32] H. Calen, J. Dyring, K. Fransson, L. Gustafsson, S. Haeggstroem, B. Hoeistad, A. Johansson and T. Johansson et al., Phys. Rev. Lett. 79 (1997) 2642.

[33] R. Bilger, W. Brodowski, H. Calen, H. Clement, C. Ekstrom, G. Faldt, K. Fransson and L. Gustafsson et al., Phys. Rev. C 69 (2004) 014003.

[34] J. Smyrski, H. -H. Adam, A. Budzanowski, E. Czerwinski, R. Czyzykiewicz, D. Gil, D. Grzonka and M. Janusz et al., Phys. Lett. B 649 (2007) 258

[35] H. -H. Adam, I. Geck, A. Khoukaz, T. Lister, R. Santo, S. Steltenkamp, A. Taschner and E. Czerwinski et al., Phys. Rev. C 75 (2007) 014004.

[36] T. Mersmann, A. Khoukaz, M. Buscher, D. Chiladze, S. Dymov, M. Hartmann, V. Hejny and A. Kacharava et al., Phys. Rev. Lett. 98 (2007) 242301

[37] F. Pheron, J. Ahrens, J. R. M. Annand, H. J. Arends, K. Bantawa, P. A. Bartolome, R. Beck and V. Bekrenev et al., Phys. Lett. B 709 (2012) 21.

[38] W. Krzemien, P. Moskal, J. Smyrski and M. Skurzok, EPJ Web Conf. 37 (2012) 02003

[39] V. A. Baskov, A. V. Koltsov, A. I. L'vov, A. I. Lebedev, L. N. Pavlyuchenko, V. V. Polyanskiy, E. V. Rzhanov and S. S. Sidorin et al., arXiv:1212.6313 [nucl-ex].

[40] C. Wilkin, M. Buscher, D. Chiladze, S. Dymov, C. Hanhart, M. Hartmann, V. Hejny and A. Kacharava et al., Phys. Lett. B 654 (2007) 92.

[41] J. A. Niskanen and H. Machner, arXiv:1207.3210 [nucl-th].

[42] K. M. Watson, Phys. Rev. 88 (1952) 1163.

[43] A. B. Migdal, Soviet Phys. JETP 1 (1955) 2; see also Qualitative methods in Quamtum theory, Perseus Cambridge MA (2000).

[44] R. Machleidt, K. Holinde and C. Elster, Phys. Rep. 149, 1 (1987).

[45] V. Baru, J. Haidenbauer, C. Hanhart and J. A. Niskanen, Eur. Phys. J. A 16 (2003) 437 nucl-th/0207040.

[46] H. S. Sherif, M. S. Abdel-monem and R. S. Sloboda, Phys. Rev. C 27, 2759 (1983).

[47] C. Lazard and Z. Maric, Nuovo Cim. A 16 (1973) 605.

[48] C. B. Dover and S. N. Yang, Phys. Lett. B 50 (1974) 217.

[49] S. Fernbach, R. Serber, and T. B. Taylor, Phys. Rev. 75, 1352 (1949).

[50] C. A. Engelbrecht, Phys. Rev. 133 (1964) B988.

[51] D. Drechsel, S. S. Kamalov and L. Tiator, Eur. Phys. J. A 34 (2007) 69 arXiv:0710.0306 [nucl-th]].

[52] B. Krusche, M. Fuchs, V. Metag, M. Robig-Landau, H. Stroher, R. Beck, F. Harter and S. J. Hall et al., Eur. Phys. J. A 6 (1999) 309.

[53] F. Rambo, P. Achenbach, J. Ahrens, H. J. Arends, R. Beck, S. J. Hall, V. Hejny and P. Jennewein et al., Nucl. Phys. A 660 (1999) 69 nucl-ex/9907020].

[54] C. Wilkin, Phys. Rev. C 47 (1993) 938 nucl-th/9301006.

[55] A. M. Green and S. Wycech, Phys. Rev. C 55, R2167 (1997).

[56] R. A. Arndt, W. J. Briscoe, T. W. Morrison, I. I. Strakovsky, R. L. Workman and A. B. Gridnev, Phys. Rev. C 72, 045202 (2005).

[57] E. P. Wigner, Phys. Rev. 98 (1955) 145.

[58] B. Mayer, A. Boudard, B. Fabbro, M. Garcon, C. Kerboul, J. Poitou, F. Wellers and W. W. Jacobs et al., Phys. Rev. C 53 (1996) 2068.

[59] H. C. Chiang, E. Oset and L. C. Liu, Phys. Rev. C 44 (1991) 738.

[60] Y. Maghrbi, B. Krusche, J. Ahrens, J. R. M. Annand, H. J. Arends, R. Beck, V. Bekrenev, B. Boillat et al., Eur. Phys. J. A 49 (2013) 38 arXiv:1303.2254 [nucl-ex]].

[61] U. Tengblad, G. Faldt and C. Wilkin, Eur. Phys. J. A 25 (2005) 267

[62] Y. N. Uzikov, Nucl. Phys. A 801 (2008) 114 arXiv:0801.0118 [nucl-th]]. 